\title{
The Reform of Publishing Format and the Reconstruction of Editing Power in the Era of Intelligence
}

\author{
Yuan Chen \\ Editorial Department of Journal of Distance Education, Zhejiang Radio and TV University, Hangzhou, China \\ Email: chy0501@126.com
}

How to cite this paper: Chen, Y. (2020) The Reform of Publishing Format and the Reconstruction of Editing Power in the Era of Intelligence. Open Access Library Journal, 7: e7052.

https://doi.org/10.4236/oalib.1107052

Received: November 30, 2020

Accepted: December 28, 2020

Published: December 31, 2020

Copyright () 2020 by author(s) and Open Access Library Inc.

This work is licensed under the Creative Commons Attribution International License (CC BY 4.0).

http://creativecommons.org/licenses/by/4.0/

\begin{abstract}
Intelligence represents the future direction of media development. The combination of intelligent technology and publishing media brings penetration and deep application, which makes the content production from the original manual process into a highly automated machine workflow. This eliminates the production relations and inherent boundaries of the traditional publishing field, changes the thinking mode and working mode of the relevant subjects in the original publishing ecological chain, and reshapes the publishing process and industrial ecology. Under the background of integrating new demands, new formats, new technologies and new talents, the prospect and analysis of publishing formats in the intelligent era and the establishment of an analysis framework of editorial power in the intelligent era are helpful for us to grasp the development trend of intelligent publishing and build an intelligent publishing format in line with ecological development. Therefore, starting from the future intelligent publishing scene, this paper discusses the reconstruction and reconstruction of the publishing content value by media intelligence, expounds the concept of editing power in the intelligent era, and from the development trend of publishing formats in the intelligent era, the ability development of editors, the main body of publishing industry, is viewed. The research shows that: the editing work in the intelligent era is not only limited to editing and proofreading work, but also closely linked with the times and integrated with the development of technology; the editing ability in the intelligent era is composed of learning ability, new human-computer cooperation quality and competency of intelligent work field; editors in intelligent era must develop their own core literacy to improve digital students in intelligent era.
\end{abstract}

\section{Subject Areas}

Journalism and Communication 


\section{Keywords}

Intelligent Era, Editing Power, Publishing Format, Digital Viability

\section{Introduction}

Intelligent technology is deeply affecting people's life, such as learning, reading, medical treatment, communication, consumption, leisure and so on. Intelligent applications such as robot, machine learning, intelligent assistant and intelligent partner are subverting and redefining the working form. According to the report "future work and vocational training" released by Pew Research Center, the work field in the era of artificial intelligence will be highly automated and systematic, and will develop into two major trends: the emergence of "cloud labor" and the replacement of artificial labor by machines [1]. Driven by technological innovation, a new work form and field is coming to us.

“2016-2017 annual report of China's digital publishing industry" points out from the perspective of publishing format that artificial intelligence will bring more possibilities for the transformation and integration of the press and publishing industry, while artificial intelligence technology will reshape the publishing process and make the publishing process intelligent. It can be predicted that in the future, as the direct beneficiary of digital publishing technology in the era of big data, the publishing industry will achieve a revolutionary breakthrough with the development of artificial intelligence and create a new era of intelligent publishing with "intelligent editing" as its main connotation.

With the penetration of intelligent media and technology integration, the research on publishing format in the intelligent era is becoming more and more heated, and the views are also more similar. For example, Wang Xiaoguang [2] looked forward to the revolutionary changes brought by artificial intelligence to the publishing industry, and discussed the potential significant impact of intelligent understanding, processing and operation of publishing content and publishing from three aspects of content creation, content editing and content distribution. Wang Heyuan [3] held a positive attitude towards the updating of publishing content, the transformation of editing functions and the industrial upgrading of the whole publishing industry.

Xu Fang [4] believes that intelligent technology has a profound impact on the paradigm process of traditional publishing industry, and from the perspective of accurate topic selection in content planning and efficient collaborative production mode, This paper expounds the Framework Reconstruction in the future publishing industry from the following aspects: intensive and efficient content editing, intelligent and fast production channel, intelligent printing of content publishing and printing, accurate push marketing mode, personalized customization of content distribution and dissemination, and reading market of interactive reading. It can be seen that the existing research on the integration of artifi- 
cial intelligence and publishing industry has a clear positioning, and a clear understanding of publishing ethics game brought by intelligent technology.

Although intelligent technology has stimulated the subversive change of publishing format, its emergence, development and application have to go through a long process of being accepted and recognized by the market [5], which still needs to be tested in practice. This paper further believes that the core of the restructuring of publishing formats in the intelligent era is content production, and the transformation and upgrading of publishing industry around the algorithm revolution in the field of content production. Therefore, this paper starts from the intelligent scene of future publishing, discusses the reconstruction of publishing content value and media integration by media intellectualization, and from the development of publishing formats in the intelligent era, the development of editors, the main body of publishing industry, can be seen through.

\section{The Reconstruction of Publishing Format by Media Intelligence}

Intelligence represents the development trend and direction of future media [6]. The rapid development of mobile Internet, supercomputer, blockchain, virtual reality, artificial intelligence, big data, 5G and other new technologies, as well as the penetration and deep application generated by the combination with publishing media, have transformed the content production from the original manual process to the highly automated machine workflow, reshaped the publishing process and format, and changed the production relationship in the publishing ecological field. In short, intelligent technology, with its advantages of computing speed, repeatability and scalability, covers most human intensive projects, which brings large-scale automation and unparalleled efficiency, and makes an unprecedented breakthrough in the depth and breadth of publishing communication.

It can be said that intelligent media eliminates the inherent boundaries of traditional publishing media and reshapes the ecology of publishing media industry, which is reflected in the whole production process of publishing content.

\subsection{Intelligent Aggregation of Content}

In the traditional media and early Internet era, the selection of unstructured text, image, audio, video and other information data depends on manual editing. However, with the precision of artificial intelligence and machine learning algorithms, especially deep learning algorithms, which have reached the level equivalent to that of human beings, many tasks can be realized automatically. For example, intelligent chemical technology can automatically insight into mining materials, realize cross platform, online connection, offline open and circular production mode, aggregate the contents of different platforms, and get accurate from massive data It can mine new content accurately and quickly, collect and organize the content, and provide continuous content production for traditional 
media with its high efficiency and aggregation characteristics. At the same time, people can also obtain important insights from these data assets and explore deeper causality.

For example, using data analysis and artificial intelligence technology to establish a database, and on this basis formed the promotion and tracking of this topic. In addition, intelligent technology can capture high-quality audio, video, pictures and other information materials, realize the integration of multiple media display modes, make the content dissemination more on-site and enhance the expression. From an editorial point of view. Book information, author information, sales information, evaluation information, reader information, etc. all use big data to make topic selection decisions and improve the ability to refine the content.

According to the frequency and popularity of major events, hot people and hot topics on the Internet, some foreign publishing institutions have carried out intelligent analysis on book topics. The New York Times disclosed to the outside world that one of its chief editors is actually a chat robot working 24 hours a day [7]. The average reading volume of selected articles edited by this machine is 38 times that of ordinary articles. AI has started with writing simple news articles and trying to write in-depth articles, even theoretical articles. In some media, speech input, machine proofreading and machine writing have become the norm.

Even in the reports of research institutions such as Yale University and Oxford University, artificial intelligence is expected to surpass human beings in translation and high school writing in the next 10 years; Large publishers like Grand Central publishing in the United States have also begun to use automatic text analysis technology, in order to assist authors in creating, even in machine creation.

\subsection{Intelligent Modeling of Content}

On the basis of information and data technology, with the help of deep learning algorithm, we can learn and analyze the subtle behavior of users. With intelligent scene matching as the feature, content, user data and services as the core resources, we can optimize the accurate configuration of supply-demand relationship between content and users based on scene data. For example, according to the user's drag and play block and the user's stay time in different video scenarios, the user's evaluation of media content such as pictures, plots, narratives and shots can be extracted for in-depth analysis, and then the media content preference can be described vertically according to the characteristics of the users. The online media transmission performance can be automatically adjusted according to the network congestion, infrastructure and bandwidth Width and other details are optimized to determine the compression level required for playback.

Through video analysis, image recognition, face recognition, language transla- 
tion, speech synthesis, virtual customer service, natural language processing and other technologies, the video, image, voice, text and other contents are transformed into media product forms suitable for various communication scenarios. In the field of augmented reality and virtual simulation, intelligent modeling is used to improve the presence of content. Some publishers are investigating whether artificial intelligence can help peer review, according to CNN. Isabelle Augenstein, a postdoctoral fellow at University College London, is working with Elsevier to develop peer-reviewed computing tools, including the selection of appropriate reviewers based on keywords in manuscripts.

Elsevier has a tool called evise, which can check plagiarism, assess reviewers' conflicts of interest, and regulate the workflow among editors, reviewers and researchers, according to wired. At the same time, spring feature is experimenting with a software tool called stat reviewer to evaluate the completion and accuracy statistics of submitted manuscripts [8].

\subsection{Accurate Dissemination of Content}

With the outbreak of network information, the media form in the network age presents the characteristics of integration, fragmentation and socialization. A large amount of information about people's activities is recorded and digitized, and then spread through social networks and social media, thus forming ubiquitous social networks and evolving. The forward-looking technology with knowledge computing engine as the core has further accelerated the development of social media. Under intelligent technology, everything is media, with the help of massive data resources, fully integrates mass communication, group communication, organizational communication, community communication and interpersonal communication.

The intelligent technology further takes the scenario as the demand interface, forms personalized recommendation according to the user's attributes, behaviors, preferences and other audience characteristics [9]. Through the visual tracking of information dissemination, it can observe the propagation path, location, etc., subdivide the crowd, enrich the user portraits, and use the perceptual intelligence to judge the state and needs of people in specific scenes On the basis of this, it can push users to meet their needs, so as to achieve personalized demand matching and targeted product push, and expand the accuracy of social communication. Then, timely obtain the feedback and evaluation data of the content for further accurate delivery. It can be said that once the publishing content is connected to the Internet, it becomes the same network existence as the website, database, map and other applications, and has a specific space-time relationship with users.

To sum up, based on the process of data acquisition, data analysis, database collation and intelligent modeling, the integration and application of intelligent technology and content production in publishing industry is constantly improving and maturing. With the support of intelligent technology, the acquisi- 
tion and collation of information-based content data will be a complete transformation of the work mode process. In the future, it will also form a content production form supported by a huge database, integrated paper media, digital media, interactive experience, and combined with the mass flow communication mode.

\section{Reconstruction of Editing Power in the Era of Intelligent Media}

\subsection{New Connotation of Editing Work in Intelligent Era}

The integration of intelligent technology and publishing industry not only reshapes the publishing process and format, but also changes the thinking mode and working mode of relevant subjects in the original publishing ecological chain. At present, publishing and editing is in the eve of great changes. On the one hand, the rapid development of network and information technology has brought great impact and challenge to the traditional editing business; on the other hand, intelligent technology has quietly penetrated into the form and structure of editing business. Editing work is not only limited to "editing" and "proofreading", but also endowed with more new connotations, closely linked with the times and integrated with the development of technology.

First of all, the emergence of public intelligent platform supporting editing has broken the traditional closed publishing platform mode, and the work form relying on the situation of "cloud labor" will appear widely. "Cloud labor" is similar to the "micro tasks" released on a platform called "universal human relationship system" operated by Microsoft. The network platform can quickly supply work resources according to labor demand; labor can choose jobs according to their own skills. In the future, the working mode of editors will change from simple desk work to creative work. As an independent module, editors provide the readers with the interactive content products they need. The public intelligent platform intelligentizes the basic work of collecting and sorting text and content resources, while editors focus on developing creativity around the content, forming a content production mode with planners as the center and public intelligent platform as the auxiliary.

Secondly, the roles of creators, consumers and processors often change, and the role of editors will be more diversified. On the one hand, the dominant power of information has changed from centralization to differentiation, and the audience's subjective consciousness in content production and dissemination has been strengthened. Including professional media production team, we media, the public, grassroots individuals can express their views from their own perspective through the network. On the other hand, editing and topic selection in the traditional sense will become the basic work for the extensive integration of knowledge resources. Big data here is not huge customer behavior data, but large content resource data and intelligent interaction with users. Editors will become the designers of specific service projects and product developers connecting 
readers and content providers.

\subsection{The Composition of Editing Power in the Era of Intelligence}

At that time, the editing tasks in the publishing process can also be undertaken by manual editing and intelligent editing respectively. Whether artificial editing will be replaced by artificial intelligence and increasingly marginalized, or completely control artificial intelligence to form a new editing form of human-computer cooperation supported by artificial intelligence technology depends on how we apply the existing technology and how to build the editing power in the intelligent era. Therefore, editors in the intelligent era must cultivate their own skills and abilities in line with the times: learning ability in the intelligent era, competence in intelligent work field and new literacy in interpersonal cooperation.

\subsubsection{Learning Power in the Era of Intelligence}

The essence of intelligence refers to the information processing and computing ability of the brain. Artificial intelligence only shares part of the memory task in the process of human intelligence operation. Now it can participate in people's thinking process by undertaking part of the logical operation task. However, artificial intelligence can't match or surpass human's thinking and consciousness as a whole. And in this era of rapid development of intelligent penetration, the past static social form has been replaced by today's dynamic social form. In the information age, with the explosive growth and rapid change of knowledge, any individual can only have a small part of knowledge. Knowledge is scattered in various nodes of the network, and can be quickly obtained through network retrieval or other ways at any time.

Therefore, only by "learning to learn" and "continuous learning", can human beings deal with such a huge knowledge system and effectively respond to the demands of the development of the times. As learning individuals, what we need to do is to connect the neural network in our brain with the external network of the Internet, constantly search, select, communicate, think and write, and become a part of the "Super Brain" of the network.

\subsubsection{New Capabilities of Intelligent Workplace}

In the intelligent workplace, editors can use AI technology, big data analysis and "cloud technology", VR, AR, etc., and artificial intelligence technologies virtual reality (VR) and augmented reality (AR) can complete the relevant work responsibilities of problem handling, communication and creation in the virtual network world. Compared with the traditional editing and proofreading work, editors need to do more creative and innovative work. Therefore, editors should not only have information literacy, information processing and network connection, cross-border knowledge and cross-border ability, but also constantly explore new fields, understand new ideas and understand new knowledge.

For example, in the process of realizing intelligence, all employees are faced 
with arduous learning tasks. We should adhere to the dynamic tracking of the latest research achievements in the basic theories of artificial intelligence, such as big data intelligence theory, cross media perception and computing theory, hybrid enhanced intelligence theory, group intelligence theory, autonomous collaborative control and optimization decision theory, etc.; we should adhere to the dynamic tracking of the latest research results in the basic theories of artificial intelligence, such as big data intelligence theory, cross media perception and computing theory, hybrid enhanced intelligence theory, group intelligence theory, autonomous collaborative control and optimization decision theory It is necessary to pay close attention to the research and development achievements of key technologies of swarm intelligence, intelligent modeling technology of virtual reality and natural language processing technology.

\subsubsection{The New Quality of Man-Machine Cooperation}

In the intelligent era, there will be many editing jobs to be replaced by machines in the future. Because the editing business has a relatively direct demand for the ability of memory, information processing and logical operation, it is necessary for artificial intelligence to partially replace or complete the relevant editing business. There is a competitive relationship between $\mathrm{AI}$ and editing, but there is also a complementary relationship. Zhang Jiong and Wu Ping [10] think that the human-computer interaction editing power is based on the traditional editing power to reposition the editor's role from the aspects of knowledge, skills, consciousness, cognition, quality and norms, so as to urge editors to transform their ideas into the "value" system of intelligent editing and the "rules" of intelligent publishing operation.

In the era of intelligence, facing the new environment brought about by intelligent technology, editors need to reconstruct their own work functions and values: to master intelligent technology, liberate themselves from complicated basic work, and compare depth and creativity with robots. Editors need to innovate in their major. On the one hand, they should construct "intelligent editing" with intelligent activities, that is, perception, understanding, identification, memory, reasoning, calculation, association, anticipation, planning and other intellectual labor, and play a leading role in the aspects of gatekeeper, leader and intervention; on the other hand, editors should master the intelligent thinking mode and operation method, and integrate personal value, self-awareness and personality characteristics and intelligent technology are combined to form human-computer interaction cooperation mechanism to complete each complex task and creative work.

\section{Summary}

The penetration of intelligent technology in the field of publishing and editing is the inevitable trend of the development of publishing and editing industry. In the future, the core work of publishing and editing is still to grasp and innovate the content. With the high efficiency given by intelligent technology, the task of 
editing will focus on providing innovative content that conforms to different media characteristics. It can be seen that the reconstruction of publishing formats focusing on content production is not only the internal requirement of the transformation and upgrading of publishing industry, but also the basic logical framework for the improvement of editors' editing power. Therefore, this paper expounds the reconstruction and reconstruction of publishing content value by media intellectualization from the aspects of intelligent gathering, intelligent customization and accurate distribution of content, and based on this, discusses the requirements of future editor training.

The integration and development of intelligent technology and publishing industry is the process of comprehensive development of technology, talents, mechanism and other factors. First of all, we should solve the ethical problems of information dissemination under intelligent technology, and cannot lose the guidance of basic values. Only by guiding the intelligent technology to operate ethically on the basis of formulating relevant laws and regulations, can we build an ecological system combining intelligent technology with editing business in the process of integration and development of intelligent technology and publishing. In this paper, there are still some deficiencies in the thinking of the ecological system of collaborative development of intelligent technology, intelligent talents and related norms. At this time, the focus of the current industry is also the focus of future publishing research, which needs to be promoted in the future.

\section{Conflicts of Interest}

The author declares no conflicts of interest regarding the publication of this paper.

\section{Fund Project}

This paper is the research results of Zhejiang Radio and TV University project "Research on multidimensional knowledge patterns and academic media innovation in 5G era (XKT-19G14); research results of 2020 scientific research project" reconstruction of academic journals' credibility from the perspective of Omnimedia (ZGXB202001) "by Zhejiang Provincial Journal Association, Zhejiang Higher Education Association".

\section{References}

[1] Pan, T.J. and Ouyang, Z.M. (2018) Work and Vocational Training in the Era of Artificial Intelligence: Development Trend and Countermeasures-Based on the Interpretation of the Future of Work and Vocational Training and Cloud Labor. Journal of Distance Education, 1, 18-26.

[2] Wang, X.G. (2017) Artificial Intelligence: The Possibility of Publishing in the Future-Looking Forward to the Future of Artificial Intelligence and Publishing. Science and Technology and Publishing, 11, 3-6.

[3] Wang, H.Y. (2018) Preliminary Study on the Reform of Publishing Industry in the 
Era of Artificial Intelligence. Communication Research, 2, 148-149.

[4] Xu, F. (2018) Reconstruction of Publishing Industry in the Era of Artificial Intelligence and Big Data. Journal of Beijing printing University, 6, 40-44.

[5] Wu, F.F. (2018) The Integration of Artificial Intelligence Technology and Publishing Industry. Publishing Wide Angle, 1, 26-28.

[6] Zhang, J.Y. (2019) New Era of Artificial Intelligence. Tsinghua University Press, Beijing.

[7] Robot Grabs the Job, the Editor in Chief of the New York Times Is Actually a Chat Robot. [2015-08-17]. [2020-05-26]. http://www.xinhuanet.com/world/2015-08/17/c_128136851.htm

[8] AI Is Expected to Be Used to Assist Peer Review. [2017-02-23]. [2017-05-26] http://www.analysis.bio/2017/02/23/ai-peer-review/

[9] Cao, H.F. (2018) Innovation and Thinking on Talent Training Mode of Editing and Publishing Specialty in the Era of Algorithm. China Editor, 9, 36-39.

[10] Zhang, J. and Wu, P. (2020) Reconstruction and Generation Path of Editorial Power System in the Era of Artificial Intelligence. Publishing Research, 4, 74-79. 